\title{
TEM Study of the Morphology and Assembly of Water-Soluble Molecular Rods
}

\author{
C. Gothard*, W.-A. Chiou ${ }^{* *}$, and J. S. Nowick ${ }^{*}$ \\ * Department of Chemistry, University of California, Irvine, CA 92697 \\ ** Dept. of Chemical Engineering and Materials Science, University of California, Irvine, CA 92697
}

Nanometer-sized building blocks provide an attractive approach to creating well-defined supramolecular architectures that may be useful in nanotechnology and nanomedicine [1]. This paper introduces a set of new building blocks that can be easily assembled into water-soluble molecular rods and macrocycles of defined and precise size and constitution. These building blocks offer a unique combination of water solubility, giant size, well-defined structure, and easy synthesis that has not been previously reported [2-3]. The purpose of this study is to gain insight into the structure and supramolecular assembly of a new class of organic semi-rigid molecular rods.

Abc (aminobiphenylcarboxylic acid) building blocks contain an amino and a carboxylic acid group, which can be coupled together using standard solid-phase peptide methodologies to form oligomers (Fig. 1). The building blocks are efficiently synthesized by way of the Suzuki cross coupling reaction, which provides the appropriately substituted biphenyl "main chain". Two alkoxyammonium side chains, designated $2 K$ due to their resemblance to the side chain of lysine, impart good water solubility to $\mathrm{Abc}^{2 \mathrm{~K}}$ oligomers. The protected version of $\mathrm{Abc}^{2 \mathrm{~K}}$, Fmoc-Abc ${ }^{2 \mathrm{~K}(\mathrm{Boc})}$ $\mathrm{OH}$, can be used to produce $\mathrm{Abc}^{2 \mathrm{~K}}$ oligomers in 1-nm increments to any defined length (e.g., 2-10 $\mathrm{nm})$. Samples of $\mathrm{Abc}^{2 \mathrm{~K}}$ octamer, $\mathrm{H}-\left(\mathrm{Abc}^{2 \mathrm{~K}}\right)_{8}-\mathrm{NH}_{2}$, were prepared by pipetting a few $\mu$ drops from aqueous solutions and dropped onto both holey carbon and SiN grids and then examined by TEM after air-drying. None of the samples were stained to enhance the contrast of the images.

A TEM micrograph of the $\mathrm{Abc}^{2 \mathrm{~K}}$ octamer $\mathrm{H}-\left(\mathrm{Abc}^{2 \mathrm{~K}}\right)_{8}-\mathrm{NH}_{2}$ (Fig. 2) shows two types of supramolecular assemblies, sheets and rings. A high-magnification TEM image of sheet structures reveals fringes that ostensibly resemble typical graphite structure (Fig. 3). The measured $0.4 \mathrm{~nm}$ separations, however, are not consistent with graphite spacing, and may reflect looser packing of the substituted aromatic rings of the $\mathrm{Abc}^{2 \mathrm{~K}}$ oligomers. Figure 4 shows a collection of $\mathrm{Abc}^{2 \mathrm{~K}}$ ring structures with diameters that range from 20 to $50 \mathrm{~nm}$. The electron diffraction depicts the somewhat ordered nanomaterials or assemblies. A close-up of a ring assembly reveals a circularly aligned pattern with a packing distance comparable to the $0.4 \mathrm{~nm}$ spacing found in sheets (Fig. 5). Figure 6 illustrates an assembly model for $\mathrm{Abc}^{2 \mathrm{~K}}$ oligomers in which the rods align to form sheets and the sheets can further assemble into circular structures that resemble rings.

A circular homologue of the oligomeric $\mathrm{Abc}^{2 \mathrm{~K}}$ rods was also prepared as a control to demonstrate that the rod shape of $\mathrm{Abc}^{2 \mathrm{~K}}$ oligomers is important for higher order assembly. A TEM image of the cyclo-octamer shows only short-range alignment and demonstrates that the cyclo-octamer cannot form sheets or other well-defined assemblies. The high resolution TEM image depicts the cyclooctamer with a morphology that resembles what might be expected from a molecular model of the well-defined macrocycle of the cyclo-oligomer (arrows in Fig. 7).

[1] http://nihroadmap.nih.gov/nanomedicine.

[2] Michl, J.; Schwab, P. F. H.; Levin, M. D. Chem. Rev., 99 (1999) 1863-1933.

[3] Tour, J. M. Chem. Rev., 96 (1996) 537-553.

[4] This research was supported by NIH Training Grant. 


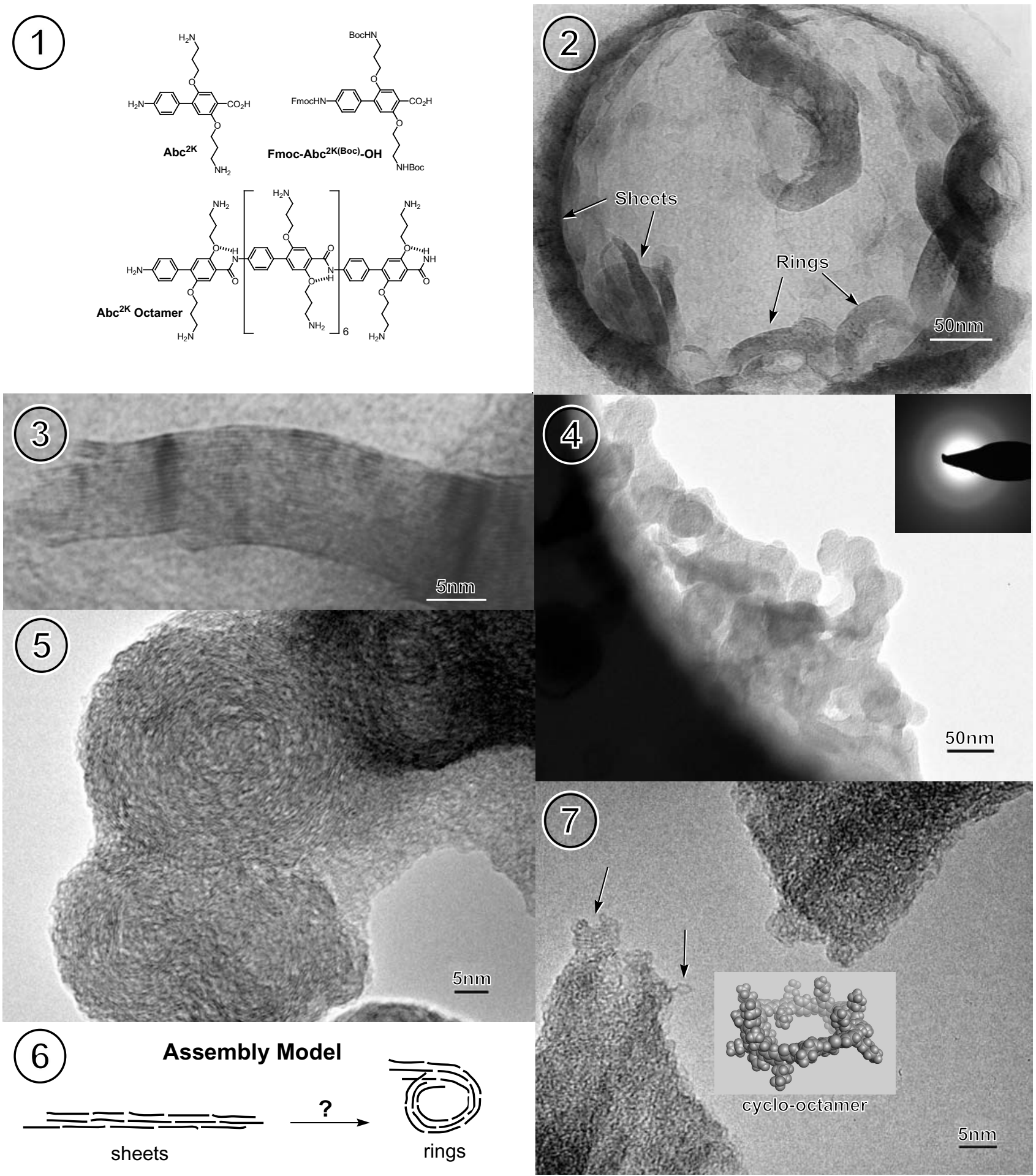

Fig. 1. Chemical structures of $\mathrm{Abc}^{2 \mathrm{~K}}$ building block, protected form, and $\mathrm{Abc}^{2 \mathrm{~K}}$ octamer.

Fig. 2. TEM micrograph revealing two types of $A \mathrm{bc}^{2 \mathrm{~K}}$ assemblies: sheets and rings.

Fig. 3. High magnification TEM image of a sheet assembly showing alignment of oligomers.

Fig. 4. Collection of circularly arranged sheets (rings) with diameters of 20-50 nm.

Fig. 5. Close-up of a ring cluster revealing fringes (ca. $0.4 \mathrm{~nm})$ aligned in a circular arrangement. Fig. 6. Assembly model illustrating oligomers aligning to form sheets before forming into rings.

Fig. 7. Control $\mathrm{Abc}^{2 \mathrm{~K}}$ cyclo-octamer showing only very short-range ordered alignment. 